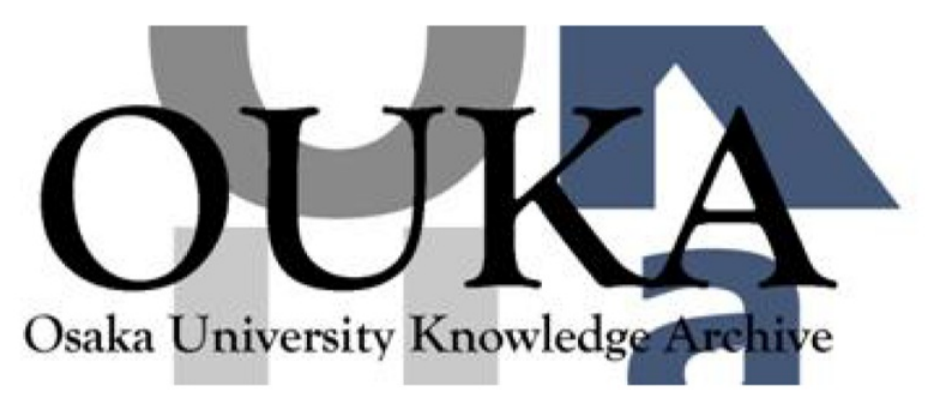

\begin{tabular}{|c|l|}
\hline Title & $\begin{array}{l}\text { Design method of dynamical decoupling sequences } \\
\text { integrated with optimal control theory }\end{array}$ \\
\hline Author(s) & $\begin{array}{l}\text { Tabuchi, Yutaka; Negoro, Makoto; Kitagawa, } \\
\text { Masahiro }\end{array}$ \\
\hline Citation & Physical Review A. 96(2) p.022331 \\
\hline Issue Date & $2017-08-31$ \\
\hline oaire:version & VoR \\
\hline URL & https://hdl.handle.net/11094/77646 \\
\hline rights & $\begin{array}{l}\text { Copyright (2017) by the American Physical } \\
\text { Society }\end{array}$ \\
\hline Note & \\
\hline
\end{tabular}

Osaka University Knowledge Archive : OUKA

https://ir. Library. osaka-u. ac. jp/

Osaka University 


\title{
Design method of dynamical decoupling sequences integrated with optimal control theory
}

\author{
Yutaka Tabuchi* \\ Research Center for Advanced Science and Technology, The University of Tokyo, Meguro-ku 153-8904, Japan \\ and Graduate School of Engineering Science, Osaka University, Toyonaka, Osaka 560-8531, Japan \\ Makoto Negoro and Masahiro Kitagawa \\ Graduate School of Engineering Science, Osaka University, Toyonaka, Osaka 560-8531, Japan
}

(Received 13 March 2016; published 31 August 2017)

\begin{abstract}
A method for synthesizing dynamical decoupling (DD) sequences is presented, which can tailor these sequences to a given set of qubits, environments, instruments, and available resources using partial information of the system. The key concept behind the generation of the DD sequences involves not only extricating the strong dependence on the coupling strengths according to the "optimal control," but also exploiting the "refocus" technique used conventionally to obtain DD sequences. The concept is a generalized one that harmonizes optimal control and designing of DD sequences.
\end{abstract}

DOI: 10.1103/PhysRevA.96.022331

\section{INTRODUCTION}

In quantum information processing (QIP), dynamical decoupling (DD) [1,2] is a useful tool for suppressing the decoherence resulting from multiple couplings among qubits and their environments. Potential applications of dynamical decoupling to quantum memory [3], quantum computation (QC) [4-7], and fault-tolerant quantum computation [8] have been proposed recently. Trains of successive pulses such as Carr-Purcell-Meiboom-Gill (CPMG), which was originally developed in the nuclear magnetic resonance (NMR) community $[9,10]$, concatenated DD, Uhrig DD (UDD), quadratic DD (QDD), and Knill DD (KDD) [3,11-13] have been proposed and demonstrated successfully to eliminate unwanted couplings [14-16] without knowledge of the surrounding environment, e.g., its coupling strengths or structures. These methods are useful for experiments in which system details are largely unavailable. Furthermore, dynamical decoupling allows us to build a quantum gate which can carry out gate operation and decouple unwanted couplings simultaneously [17-19]. This has been successfully implemented to demonstrate a quantum algorithm [20]. In practical experiments, however, theoretical approximations such as the $\delta$-pulse approximation result in the finite pulse width problem [21], and the requirement of an infinite frequency bandwidth for a rectangular pulse causes deformation at the leading and trailing edges of the pulse [22,23]. Both of these practical resource limitations cause serious bottlenecks when using pulse DD sequences. References [24,25] provide a beautiful prescription for the finite-width problem, which is derived based on dynamical symmetrization. Fast repetition to reach the bang-bang limit may require unreasonable operation in experiments, given that the way to determine subintervals is arbitrary. In contrast, application of optimal control theory (OCT) to build a quantum gate and decoupling sequence [26-28] makes use of the complete qubit- and environment-related information to avoid such bandwidth divergence. These methods are attractive [29] when one can obtain details of a qubit-bath system, although

\footnotetext{
*tabuchi@qc.rcast.u-tokyo.ac.jp
}

spectroscopy of qubits and generation of pulse sequences become difficult as the system size increases because we have to fully grasp and treat a large Hilbert space.

It would be beneficial to harmonize OCT and pulse-based DD to avoid harmful effects arising out of practical resource limitation and inevitable error. There are a few levels to harmonize OCT and DD. One of the most intuitive and simplest ways is to replace $\pi / 2$ and $\pi$ pulses in a conventional DD sequence with the shaped pulses optimized by OCT [30]. This method can be applied to almost all pulsed DD sequence. The next level of harmonization is to numerically synthesize entire DD sequences or waveforms using OCT. We would anticipate having a much tailored DD sequence for experiments. Given that the optimization process is carried out using a computer, we can flexibly impose requirements upon DD sequences, e.g., energy and bandwidth constraints. For the synthesis of a DD sequence (waveform) in OCT, we define the parameters of a DD sequence to be optimized, for example, the amplitudes and phases of the pulses, and define a cost function which evaluates the decoupling infidelity of the DD sequence. Numerical synthesis is carried out so that the cost function is minimized by optimizing the waveform parameters of the DD sequence. How the cost function is defined is important because it strongly affects the computational complexity and decoupling efficiency. Two methods using approximation to alleviate computational complexity rather than using the full unitary evolution of the quantum system have been proposed. The authors in Refs. [3133] focused on the noise spectrum of the environment for building cost functions. In their method, decoherence arising from the environmental noise can be suppressed by applying qubit operations so that the overlap between the environmental noise spectrum and the qubit modulation spectrum is reduced. Furthermore, in their example in Ref. [32], they imposed energy constraints on DD waveforms to suppress leakage to the subspaces other than the qubit space in multilevel systems and successfully demonstrated in simulation. This method is fairly helpful for atomic qubits and superconducting qubits. A similar idea has been proposed in Ref. [34], in which they apply a multifrequency sinusoidal drive to the qubit. They engineer the qubit modulation spectrum so as to avoid a single Rabi-like intense peak in the spectrum that is overlapping 
the corresponding noise component. The application of a well-designed multifrequency drive efficiently alleviates such noise overlapping. The authors in Refs. [35,36] focused on the coupling symmetries between a qubit and surrounding environment. They restricted their DD waveforms to singleaxis rotation, and optimized the time-varying amplitude of a DD waveform so that the effective Hamiltonian of the qubit-environment couplings up to the first order was averaged out. In their method, although the method is limited to single-qubit cases, we can numerically design DD sequences which decouple noises arising from the environment without knowing the exact coupling strengths to the environment. This method also maintains flexibility in OCT where we can easily impose constraints to the DD sequences.

In this paper, we extend their single-axis rotation to the rotations about time-varying arbitrary axes in the $x-y$ plane by allowing the time-varying amplitude and phase of the DD waveforms. We anticipate wide varieties of qubit control and more precise qubit decoupling with such qubit rotation around arbitrary axes with arbitrary speeds, given that the application of time-varying noncommuting operators to qubits is more general than single-axis rotations and separate application of two-axis rotations, e.g., in QDD and MREV [37]. We show in our first example that by using such arbitrary time-varying rotation, we can design a decoupling waveform which is more robust against amplitude and phase errors compared to conventional pulse-based DD sequences.

Furthermore, our method is a natural extension to multiqubit cases. Two- or multiqubit gates are essential elements in QIP experiments. To switch on-and-off interactions to make such multiqubit gates, DD would be an alternative candidate. As compared to OCT based on unitary evolutions, the proposed method helps us to alleviate computational cost to optimize the DD sequence, provided that a cycle rate of $1 / T$ in DD is much faster than the interaction strength. The large alleviation stems from the approximation based on the average Hamiltonian theory, in which the dynamics of the system can be described by a small subset of the generator space of unitary evolutions for shorter time compared to the characteristic time of the system. In our second example, we show that we can numerically synthesize a decoupling waveform which turns on and off qubit-qubit couplings and compare it with a conventional pulsed DD sequence which applies two-axis rotations separately.

The paper is organized as follows: In Sec. II, we describe the basic concept of our DD design. In Sec. III, we present a pragmatic example in which a single qubit is coupled to many two-level systems, and where the control field has imperfections due to instruments. In Sec. IV, we apply our method to a multiqubit system where the qubits couple to each other. In $\mathrm{Sec}$. V, we discuss the performance of DD sequences generated under resource restrictions in terms of energy and bandwidth. The appendices describe how the average Hamiltonians are calculated using Floquet Hamiltonian theory in the examples.

\section{BASIC CONCEPT}

Let $\hat{\mathcal{H}}_{0}$ be the Hamiltonian of a system under consideration, and $\hat{V}(t)$ be a time-dependent external perturbation, represented in an appropriate rotating frame. We use $\hbar=1$ units throughout. The system Hamiltonian $\hat{\mathcal{H}}_{0}$ can be expressed as

$$
\hat{\mathcal{H}}_{0}=\sum_{\alpha} h_{\alpha} \hat{\Theta}_{\alpha}
$$

using a set of orthonormal Hermitian operators $\left\{\hat{\Theta}_{\alpha} \mid \hat{\Theta}_{\alpha}=\right.$ $\left.\hat{\Theta}_{\alpha}^{\dagger}, \operatorname{Tr}\left[\hat{\Theta}_{\alpha} \hat{\Theta}_{\beta}\right]=\delta_{\alpha \beta}\right\}$ and their coefficients $\left\{h_{\alpha}\right\}$. For example, a system Hamiltonian that describes the coupling between a single qubit and the surrounding environment can be expanded with operators $\hat{\Theta}_{\alpha}=\frac{1}{2} \hat{\sigma}_{\alpha} \otimes \hat{B}_{\alpha}(\alpha=x, y, z)$, where $\hat{\sigma}_{\alpha}$ are the qubit's Pauli operators and $\hat{B}_{\alpha}$ are bath operators. Consider $\hat{\mathcal{H}}_{0}$ in the interaction frame of $\hat{V}(t)$ using unitary operator $\hat{U}_{V}(t)=\hat{\mathcal{T}} \exp \left[i \int_{0}^{t} \hat{V}\left(t^{\prime}\right) d t^{\prime}\right]$, where $i=\sqrt{-1}$ and $\hat{\mathcal{T}}$ is the time-ordering operator. The Hamiltonian of Eq. (1) in the frame of $\hat{V}(t)$ then becomes the modulated Hamiltonian $\tilde{\mathcal{H}}_{0}(t)$ as follows:

$$
\hat{\tilde{\mathcal{H}}}_{0}(t)=\hat{U}_{V}(t) \hat{\mathcal{H}}_{0} \hat{U}_{V}^{\dagger}(t)=\sum_{\alpha} h_{\alpha}\left(\sum_{\beta} c_{\beta \alpha}(t) \hat{\Theta}_{\beta}\right),
$$

where $\hat{U}_{V}(t) \hat{\Theta}_{\alpha} \hat{U}_{V}^{\dagger}(t)=\sum_{\beta} c_{\beta \alpha}(t) \hat{\Theta}_{\beta}$. In this process, the effect of finite pulse width is naturally incorporated into $c_{\beta \alpha}(t)$. Compared to Ref. [24] in which they do not explicitly define subintervals, application of any waveform or finite-width pulses are fully characterized by the $c_{\beta \alpha}(t)$. The $\delta$-pulse approximation of $\hat{V}(t)$ can also be treated as discontinuities of $c_{\beta \alpha}(t)$. We call $c_{\beta \alpha}(t)$ a system-modulation matrix, as used in Ref. [32].

This system can be stroboscopically "time suspended," that is, decoupled from the environment, if there is an external perturbation $\hat{V}(t)$ such that the average Hamiltonian $\hat{\mathcal{H}}_{\text {eff }}$ of $\hat{\tilde{\mathcal{H}}}_{0}(t)$ vanishes $[2,27,38]$, and if the period $T$ of $\hat{\mathcal{H}}_{\text {eff }}$ satisfies $\left\|\hat{\mathcal{H}}_{0} T\right\|<1$ so that Magnus expansion of the evolution operator converges. Suppose $\hat{\tilde{\mathcal{H}}}_{0}(t)$ has $T$ periodicity; then, the average Hamiltonians $[2,38]$ up to the first order become

$$
\hat{\mathcal{H}}_{\text {eff }}^{(0)}=\frac{1}{T} \int_{0}^{T} \hat{\tilde{\mathcal{H}}}_{0}\left(t^{\prime}\right) d t^{\prime}=\sum_{\alpha, \beta} h_{\alpha}\left(\frac{1}{T} \int_{0}^{T} c_{\beta \alpha}\left(t^{\prime}\right) d t^{\prime}\right) \hat{\Theta}_{\beta},
$$

$$
\begin{aligned}
\hat{\mathcal{H}}_{\mathrm{eff}}^{(1)}= & \frac{1}{2 i T} \sum_{\alpha, \alpha^{\prime}} h_{\alpha} h_{\alpha^{\prime}} \sum_{\beta<\beta^{\prime}}\left(\int _ { 0 } ^ { T } d t _ { 2 } \int _ { 0 } ^ { t _ { 2 } } d t _ { 1 } \left\{c_{\beta^{\prime} \alpha^{\prime}}\left(t_{2}\right) c_{\beta \alpha}\left(t_{1}\right)\right.\right. \\
& \left.\left.-c_{\beta^{\prime} \alpha}\left(t_{1}\right) c_{\beta \alpha^{\prime}}\left(t_{2}\right)\right\}\right)\left[\hat{\Theta}_{\beta^{\prime}}, \hat{\Theta}_{\beta}\right] .
\end{aligned}
$$

We can obtain DD sequences $\hat{V}(t)$ by minimizing the coefficients of the operators $\hat{\Theta}_{\beta}$ and $\left[\hat{\Theta}_{\beta^{\prime}}, \hat{\Theta}_{\beta}\right]$, i.e., the terms within the large parentheses in Eqs. (3) and (4). Given that the external perturbation $\hat{V}(t)$ does not always guarantee the $T$ periodicity of $\hat{\tilde{\mathcal{H}}}_{0}(t)$, the system-modulation matrix $c_{\beta \alpha}(t)$ should be constrained such that $c_{\beta \alpha}(t)$ are continuous at $t=0, T, \ldots$.

For numerical minimization, $\hat{V}(t)$ should be parameterized with pulse timings, flip angles, phases, and so on. When the parameters are denoted by $\zeta=\left\{\zeta_{1}, \zeta_{2}, \ldots\right\}$, the systemmodulation matrix is also parameterized such that $c_{\beta \alpha}(t)=$ $c_{\beta \alpha}(t ; \zeta)$. For example, some quantum computation schemes require a state measurement during gate operations to protect 
qubits from decaying [39]. In our method, we naturally have a measurement window that is parameterized by start and end timing in a DD sequence, given that we can choose parameters arbitrarily. The decoupling efficiency of a generated DD sequence depends on the parameters because they are directly related to control degree of freedom. It may seem that we need to carefully choose the parameters; however, they can be chosen suitably for an experiment in practice. In other words, this method provides a way to generate the almost best DD sequences within the available resources which are indicated by the parameters. In Sec. V, we will discuss how the DD efficiency improves as the resources that are available increase.

Given that a DD sequence should make its effective Hamiltonian to zero in order to protect qubits, we optimize the parameters $\zeta$ to minimize the effective Hamiltonian. If we define

$$
\begin{aligned}
\Phi_{\alpha \beta}^{(0)}(\zeta)= & T^{-1} \int_{0}^{T} c_{\beta \alpha}\left(t^{\prime} ; \zeta\right) d t^{\prime}, \\
\Phi_{\alpha^{\prime} \alpha, \beta^{\prime} \beta}^{(1)}(\zeta)= & T^{-1} \int_{0}^{T} d t_{2} \int_{0}^{t_{2}} d t_{1} \\
& \times\left\{c_{\beta^{\prime} \alpha^{\prime}}\left(t_{2} ; \zeta\right) c_{\beta \alpha}\left(t_{1} ; \zeta\right)\right. \\
& \left.-c_{\beta^{\prime} \alpha}\left(t_{1} ; \zeta\right)_{\beta \alpha^{\prime}}\left(t_{2} ; \zeta\right)\right\},
\end{aligned}
$$

the effective Hamiltonian up to the first order can be expressed as follows:

$$
\begin{aligned}
\hat{\mathcal{\mathcal { H }}}_{\text {eff }}= & \sum_{\alpha, \beta} h_{\alpha} \Phi_{\alpha \beta}^{(0)}(\zeta) \hat{\Theta}_{\beta} \\
& +\frac{1}{2 i} \sum_{\alpha, \alpha^{\prime}} h_{\alpha} h_{\alpha^{\prime}} \sum_{\beta<\beta^{\prime}} \Phi_{\alpha^{\prime} \alpha, \beta^{\prime} \beta}^{(1)}(\zeta)\left[\hat{\Theta}_{\beta^{\prime}}, \hat{\Theta}_{\beta}\right] .
\end{aligned}
$$

To minimize the effective Hamiltonian parameterized by $\zeta$, a cost function $\Phi(\zeta)$ for the numerical minimization is constructed such that the $\Phi_{\alpha \beta}^{(0)}$ and $\Phi_{\alpha^{\prime} \alpha, \beta^{\prime} \beta}^{(1)}$ show the residuals against the ideal DD operation that cancels couplings between qubits and its environment, i.e.,

$$
\Phi(\zeta)=\sum_{\alpha, \beta}\left|\Phi_{\alpha \beta}^{(0)}(\zeta)\right|^{2}+w^{2} \sum_{\alpha^{\prime} \alpha, \beta^{\prime}<\beta}\left|\Phi_{\alpha^{\prime} \alpha, \beta^{\prime} \beta}^{(1)}(\zeta)\right|^{2} .
$$

A guide for choosing a weight factor $w$ is $w=\left\|\mathcal{H}_{0} T\right\|$, given that the effects of the sth-order average Hamiltonian reduce with $w^{s}$. Here, we choose $w=1$ for simplicity. A penalty function $\Phi_{P}(\zeta)$ that imposes the continuity of $c_{\beta \alpha}(t)$ at $t=$ $0, T, \ldots$ on the DD sequence is defined as follows:

$$
\Phi_{P}(\zeta)=\sum_{\alpha, \beta}\left|c_{\beta \alpha}(T ; \zeta)-c_{\beta \alpha}(0 ; \zeta)\right|^{2} .
$$

Consideration of higher-order perturbations would result in better decoupling performance; however, doing so would involve cumbersome hand calculations for deriving average Hamiltonians.

The synthesis of DD sequences is then reduced to a nonlinear minimization problem,

$$
\min _{\zeta} \Phi(\zeta) \text { subjected to } \Phi_{P}(\zeta)=0,
$$

which can be numerically performed on a modern digital computer.
This method is basically the same as that given by Eq. (10) in [27], except that the cost function is constructed in Lie algebra. However, this difference allows us to choose among sequences of DD type, optimal control type [26-28], or a combination of both of these types. In general, the expansion coefficients $\left\{h_{\alpha}\right\}$ in Eq. (1) contain the details of the system, e.g., coupling strengths and frequency shifts, which can be obtained through spectroscopy. In the synthesis of DD-type sequences, which do not require knowledge of the qubit's surroundings, $\left\{h_{\alpha}\right\}$ should be excluded from the cost functions in Eqs. (5) and (6) so that the generated sequences do not depend on them. For the case where $\left\{h_{\alpha}\right\}$ are known, sequences can be synthesized with their help. If we explicitly include $\left\{h_{\alpha}\right\}$ in a cost function, for example, Eq. (5) becomes

$$
\Phi_{\beta}^{(0)}(\zeta)=T^{-1}\left|\sum_{\alpha} h_{\alpha} \int_{0}^{T} c_{\beta \alpha}\left(t^{\prime} ; \zeta\right) d t^{\prime}\right|^{2},
$$

and the obtained sequences suppress decoherence using that information. In the limit of $\|\hat{\mathcal{H}} T\| \rightarrow 0$ or in the limit of $n_{0} \rightarrow \infty$, where $n_{0}$ is the maximum order of an average Hamiltonian, this method corresponds to unitary-matrix-based optimal control.

Furthermore, cost functions in Lie algebra alleviate the growth of computational complexity as a system becomes larger. The key to the alleviation is an approximation using average Hamiltonian theory. Given that the effective Hamiltonian up to the first order does not require full operator bases in $\mathfrak{s u}\left(2^{n}\right)$, we can synthesize DD sequences in a small subset in $\mathfrak{s u}\left(2^{n}\right)$ for $n$ qubits. There is a dramatic decrease in computational costs associated with numerical optimization for a system Hamiltonian that is simple and highly symmetric, i.e., it is described with a small number of operators in Lie algebra. For individually controllable qubits, operator space does not grow exponentially with $n$, if the higher-order terms in the effective Hamiltonian can be safely neglected. We give a paradigmatic explanation in which a Hamiltonian $\hat{\mathcal{H}}_{0}=$ $\sum_{k=0}^{n} g^{(k)} \hat{\sigma}_{z, k}$ dictates a system, where $\sigma_{z, k}$ is the $k$ th qubit's Pauli $z$ operator, $\left\{g^{(k)}\right\}$ are inhomogeneously broadened energy shifts, and $n$ is the number of qubits. In the optimal control type, to obtain a DD sequence (time-suspension sequence), we have to compute $2^{n} \times 2^{n}$ matrices, which are intractable for large $n$. However, a well-known method that flips the sign of $\hat{\sigma}_{z, k}$ in the frame of $\hat{V}(t)$ by applying consecutive $\pi$ pulses to the qubits, such as CPMG [9], refocuses the inhomogeneity. Our method automates such a flipping procedure for a given system.

Note that while synthesizing DD sequences, $\hat{\mathcal{H}}_{0}$ should be modulated by $\hat{U}_{V}(t)$. This requirement is equivalent to the bracket generation condition mentioned in Ref. [27].

In the following two examples, we impose a bandwidth limitation to DD sequences, $\hat{V}(t)=\sum_{\alpha} v_{\alpha}(t) \hat{\Theta}_{\alpha}$. Such pulses or waveforms under the limitation are robust against shape deformation caused by the group delay dispersion of transmission lines, given that the pulses having wider bandwidth are easily degraded by the dispersion. These pulses under bandwidth limitation should have smooth envelopes. We use Fourier coefficients of

$$
v_{\alpha}(t)=\sum_{n=1}^{p} v_{\alpha, n} \sin (2 n \pi t / T)
$$


as the parameters of $\hat{V}(t)$, so that $\zeta=\left\{v_{\alpha, n}\right\}$. For simplicity, we use sine coefficients to reduce the number of parameters in the following examples. The Floquet average Hamiltonian theory [40] is useful to calculate the average Hamiltonian when a system-modulation matrix is $T$ periodic and expressed as the Fourier expansion, $c_{\beta \alpha}(t)=\sum_{n} c_{\beta \alpha, n} \exp (2 i \pi n t / T)$. A brief explanation of this calculation is given in Appendix A, and the cost functions in the following examples are built using the theory.

\section{DEPHASING PROBLEM}

Let us consider the qubit dephasing problem. A single qubit is coupled to a bath consisting of many two-level systems (TLSs) without energy relaxation. When there exist inevitable pulse errors caused by instruments, an experimenter might need a DD sequence that is robust to these errors. In this example, we show how to incorporate Tycko's composite pulses [41] into DD designing. In Ref. [41], a Hamiltonian of an external perturbation to control the qubits is described as $\hat{V}(t)+\hat{\mathcal{H}}_{E}(t)$, where $\hat{V}(t)$ denotes an ideal operation to the qubit and $\hat{\mathcal{H}}_{E}(t)$ represents systematic errors due to the instruments. Self-compensating composite pulses are built so that the ideal pulsing of $\hat{V}(t)$ decouples error Hamiltonian $\hat{\mathcal{H}}_{E}(t)$.

Let the Hamiltonian of the system be $\hat{\mathcal{H}}_{0}=\hat{\mathcal{H}}_{Q-B}+$ $\hat{\mathcal{H}}_{E}(t)$ and the ideal external perturbation be $\hat{V}(t)=$ $v_{x}(t) \hat{\sigma}_{x}+v_{y}(t) \hat{\sigma}_{y}$, where the qubit-bath interaction is $\hat{\mathcal{H}}_{Q-B}=$ $\sum_{k} g^{(k)} \hat{\sigma}_{z} \hat{\sigma}_{z, k}$ and the pulse error term is $\hat{\mathcal{H}}_{E}=\varepsilon_{1} v_{x}(t) \hat{\sigma}_{x}+$ $\varepsilon_{3} v_{y}(t) \hat{\sigma}_{y}+\varepsilon_{2} v_{y}(t) \hat{\sigma}_{x}+\varepsilon_{4} v_{x}(t) \hat{\sigma}_{y}$. Here, $\hat{\sigma}_{\alpha}$ and $\hat{\sigma}_{\alpha, k}$ denote Pauli operators of the qubit and the $k$ th TLS, respectively. $\varepsilon_{1}$ and $\varepsilon_{3}$ denote the error amplitudes of flip-angle error, while $\varepsilon_{2}$ and $\varepsilon_{4}$ denote the error amplitudes of phase-orthogonality error arising, for example, from microwave in-phase and quadraturephase (IQ) mixers or hybrid circuits. The external perturbation $\hat{V}(t)$ modulates the Hamiltonian $\hat{\mathcal{H}}_{0}$ in its interaction frame, so that

$$
\begin{aligned}
\hat{\tilde{\mathcal{H}}}_{0}(t)= & \sum_{k} \sum_{\beta} g^{(k)} c_{\beta z}(t) \hat{\sigma}_{\beta} \hat{\sigma}_{z, k} \\
& +\sum_{\beta}\left\{\varepsilon_{1} v_{x}(t) c_{\beta x}(t)+\varepsilon_{3} v_{y}(t) c_{\beta y}(t)\right. \\
& \left.+\varepsilon_{2} v_{y}(t) c_{\beta x}(t)+\varepsilon_{4} v_{x}(t) c_{\beta y}(t)\right\} \hat{\sigma}_{\beta},
\end{aligned}
$$

where $\sum_{\beta} c_{\beta \alpha}(t) \hat{\sigma}_{\beta}=\hat{U}_{V}(t) \hat{\sigma}_{\alpha} \hat{U}_{V}^{\dagger}(t)$. In this example, the zeroth-order cost function is defined as

$$
\Phi^{(0)}=\sum_{\beta}\left|\int_{0}^{T} c_{\beta z}(t) d t\right|^{2}+\sum_{\beta}\left|\int_{0}^{T} v_{x}(t) c_{\beta x}(t) d t\right|^{2}+\cdots,
$$

and the first-order cost function is also defined in the same manner (see Appendices B and C for detailed derivations). Exclusion of $g^{(k)}$ and $\varepsilon_{j}(j=1, \ldots, 4)$ from the overall cost function gives DD-type pulse sequences with a tolerance to pulse imperfections. DD sequences generated with this method can be tailored to the system by multiplying deliberate weights to terms in the cost function. We minimized the cost function by optimizing $\zeta=\left\{v_{\alpha, n}\right\}$ using a steepestdescent method in combination with a genetic algorithm.
TABLE I. Parameters of the DD sequence generated in Sec. III.

\begin{tabular}{lcr}
\hline \hline$n$ & $v_{x, n} T / \pi$ & \multicolumn{1}{c}{$v_{y, n} T / \pi$} \\
\hline 1 & -0.7030256 & -3.6201768 \\
2 & 3.3281747 & 3.8753985 \\
3 & 11.390077 & -1.2311919 \\
4 & 2.9375301 & -0.2998110 \\
5 & -1.8758792 & 3.1170274 \\
6 & 1.7478474 & 0.3956137 \\
7 & 5.6966577 & -0.3593987 \\
8 & -0.5452435 & -3.5266063 \\
9 & 4.0826786 & 2.4900307 \\
\hline \hline
\end{tabular}

The generated waveform expressed in Fourier series form, $v_{\alpha}(t)=\sum_{n=1}^{9} v_{\alpha, n} \sin (2 \pi n t / T)$, as listed in Table $\mathrm{I}$, is shown in the top panel of Fig. 1.

We evaluated the performance of our synthesized DD sequence, $\mathrm{UDD}_{12}, \mathrm{QDD}_{3}$, and $\mathrm{KDD}$ against the flip-angle error $\Delta \beta=\varepsilon_{1}=\varepsilon_{3}$ and the phase-orthogonality error $\Delta \varphi=$ $\tan ^{-1}\left[\varepsilon_{2} v_{y}(t) / v_{x}(t)\right]$ on a single-qubit system coupled to four bath TLSs, with the fidelity of quantum gate $\mathcal{C}$ defined as

$$
F(\mathcal{C})=\min _{|\phi\rangle}\|\sqrt{|\phi\rangle\langle\phi|} \sqrt{\mathcal{C}(|\phi\rangle\langle\phi|)}\|_{\text {tr }} .
$$

We have chosen $\varepsilon_{4}=0$. The number of pulses and the pulse widths in UDD and QDD sequences were chosen
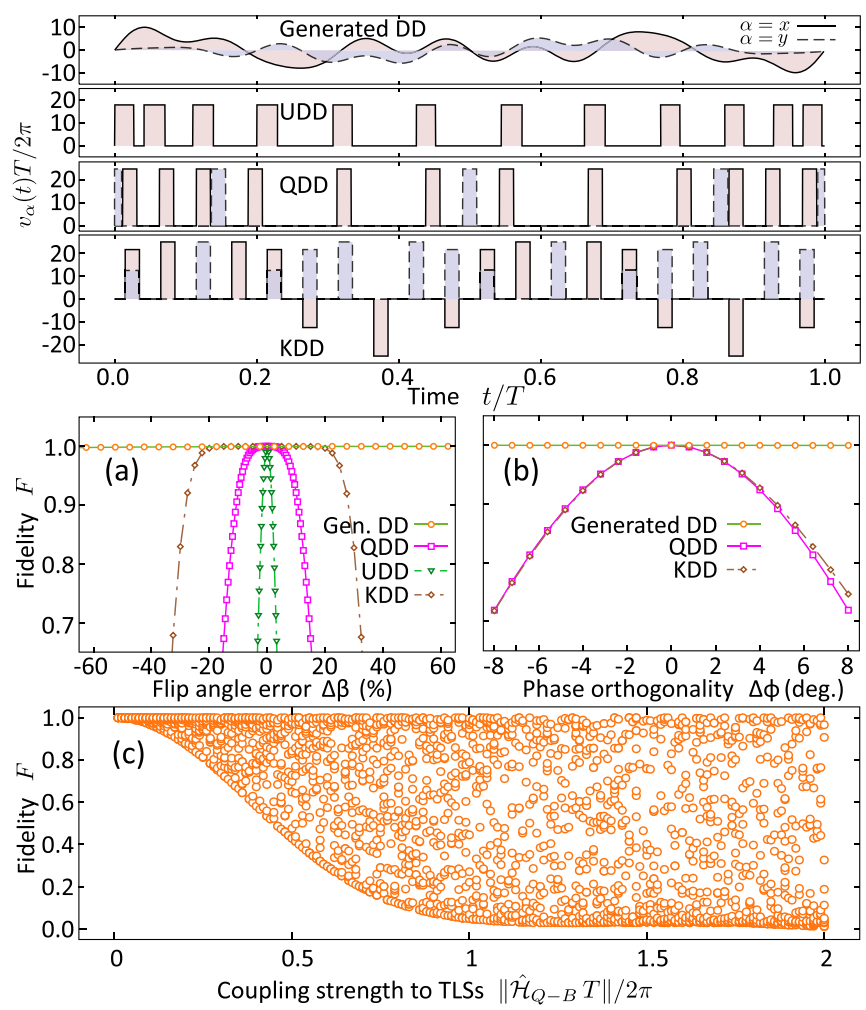

FIG. 1. Decoupling fidelities against (a) flip-angle error and (b) phase-orthogonality error for a single qubit coupled to four bath TLSs with $\mathrm{UDD}_{12}, \mathrm{QDD}_{3}$, and $\mathrm{KDD}$ sequences, and the sequence generated in this example. The waveforms of these sequences are displayed in the top panel of the figure. (c) Decoupling fidelities against coupling strengths to four TLSs denoted by $\left\|\hat{\mathcal{H}}_{Q-B}\right\|$. Coupling strengths to TLSs were randomly generated for each point in the graph. 
such that the total amounts of the applied energy and the peak amplitudes were nearly equal to those of the generated waveform. If the energy of a DD sequence is defined as $\sqrt{\int_{0}^{T} \sum_{\alpha=x, y} v_{\alpha}^{2}(t) d t}$, the energies per cycle of the generated DD sequence, $\mathrm{UDD}_{12}, \mathrm{QDD}_{3}$, and $\mathrm{KDD}$ amount to $11.50 \pi / T$, $12 \pi / T, 16 \pi / T$, and $20 \pi / T$, respectively. The peak amplitudes $\max _{t \in[0, T]} \sqrt{\sum_{\alpha} v_{\alpha}^{2}(t)} T / 2 \pi$ of the DD sequences were limited to $10,20,25$, and 25 for the synthesized DD waveform, $\mathrm{UDD}_{12}, \mathrm{QDD}_{3}$, and $\mathrm{KDD}$, respectively. Given that there is minimal interpulse spacing in UDD and QDD, their peak amplitudes were not equal to that of the synthesized DD sequence. The coupling strengths between the qubit and TLSs were randomly generated; we used $\left\{g^{(k)} T / \pi\right\}_{k=1}^{4}=$ $\{0.1353055,-0.3625386,0.0057979,0.2963580\}$, so that $\left\|\hat{\mathcal{H}}_{0} T\right\| / 2 \pi \sim 0.1$.

As the flip-angle errors are accumulated at each $\pi$ pulse, UDD is sensitive to the flip-angle error [Fig. 1(a)]; on the other hand, QDD cancels the flip-angle error by inserting a $90^{\circ}$ phase-shifted $\pi$ pulse. However, as seen from Fig. 1(b), QDD cannot compensate for the phase-orthogonality error. The KDD pulse sequence shown in the third row of the top panel in Fig. 1 has pulses with jumping phases with constant amplitude, which is intended to compensate flip-angle errors. The performance is far superior to $\mathrm{UDD}_{12}$ and $\mathrm{QDD}_{3}$ for flip-angle errors, having similar robustness to $\mathrm{QDD}_{3}$ against phase-orthogonality error. Figures 1(a) and 1(b) show that the generated sequence is robust against the pulse errors caused by the instruments. This example shows that a DD sequence can be optimized to be robust against known systematic errors, which are small but accumulate after millions of cycles in actual experiments. A variation of this example, where there is crosstalk between control pulses, can be managed in the same manner. Another variation is that the behavior of the surrounding bath TLSs is known, the Hamiltonian of which is denoted by $\hat{\mathcal{H}}_{B}$, e.g., the principal axes of the TLSs are tilted from the $z$ axis so that $\left[\hat{\mathcal{H}}_{Q-B}, \hat{\mathcal{H}}_{B}\right] \neq 0$. In this case, taking the bath Hamiltonian of the TLSs into account, we can obtain a DD sequence that overcomes degradation due to $\hat{\mathcal{H}}_{B}$ by minimizing higher-order cost functions $\Phi^{(n)}$, at least up to the second order.

Figure 1(c) shows fidelities against various sets of coupling strengths in order to investigate the robustness of the synthesized DD waveform. In a system where a single qubit is coupled to four TLSs, we evaluate fidelities for the sets of coupling strengths which are randomly generated. Figure 1(c) suggests the existence of the lowest bound of fidelity. For coupling strengths randomly distributed but $\left\|\hat{\mathcal{H}}_{Q-B} T\right\| \ll 1$ (high-repetition-rate regime), the DD waveform works efficiently under the approximation based on average Hamiltonian theory. Thus, Fig. 1(c) clearly indicates that OCT and DD are successfully harmonized; the weakness in OCT, i.e., a generated external perturbation is only applicable for a single set of coupling strengths, is overcome by the refocusing technique in DD, and numerical synthesis of external perturbations using OCT is safely applied to the generation of DD waveforms for $\left\|\mathcal{H}_{Q-B} T\right\| \ll 1$. The achievable fidelity should be bounded by the residuals in the cost function or the higher-order terms in the effective Hamiltonian. To obtain broader robustness against coupling strengths, we have to include the higher-order terms in the cost function $\Phi^{(n)}$. However, the DD efficiency might be degraded due to resource restrictions. We will discuss this trade-off between the achievable fidelity and the resources in Sec. V.

\section{MULTIQUBIT COUPLING}

In this section, we show that the proposed method is applicable to multiqubit cases. The dynamic control of qubitqubit interactions, which are used for realizing multiqubit gates, is an important technique in QIP experiments. Optimal control theory based on a unitary evolution is useful for optimizing such dynamic control by numerical computations. In general, however, for qubits having noncommuting twobody interactions between them, the interactions generate irreducible unitary evolutions among them. The fact that the size of unitary matrices grows exponentially with the number of qubits easily leads to intractable computational costs in OCT. Here, we show that a DD waveform can be obtained by solely considering two-qubit interactions, and it can switch off all the interactions among $n$ qubits.

Consider a system that consists of a finite number of qubits coupled to each other. Access to the qubits is assumed to be restricted to collective manipulation, the external perturbation of which is given by $\hat{V}(t)=v_{x}(t) \sum_{k} \hat{\sigma}_{x, k}+v_{y}(t) \sum_{k} \hat{\sigma}_{y, k}$. Each qubit is considered as being connected through a qubitqubit Hamiltonian,

$$
\begin{aligned}
\hat{\mathcal{H}}_{0}= & \hat{\mathcal{H}}_{Q-Q}=\sum_{k^{\prime}<k} d^{\left(k^{\prime}, k\right)} \\
& \times\left(\hat{\sigma}_{z, k^{\prime}} \hat{\sigma}_{z, k}-\hat{\sigma}_{x, k^{\prime}} \hat{\sigma}_{x, k} / 2-\hat{\sigma}_{y, k^{\prime}} \hat{\sigma}_{y, k} / 2\right) .
\end{aligned}
$$

In this example, we assume the qubit-qubit Hamiltonian is a dipolar Hamiltonian in a solid-state nuclear spin system with high-field approximation for example. Dynamic control of the qubit interactions can be used for QIP experiments [42-44]. This Hamiltonian contains $\hat{\sigma}_{x, k} \hat{\sigma}_{x, k^{\prime}}$ and $\hat{\sigma}_{z, k} \hat{\sigma}_{z, k^{\prime}}$ type interactions, which also appear in the superconducting qubit community for realizing two-qubit gates. Thus, the Hamiltonian chosen is a good example for both spin and superconducting qubit communities to control the coupling. We do not restrict the types of qubit-qubit couplings in the proposed method except that the couplings commute with external perturbations, i.e., $\left[\hat{\mathcal{H}}_{Q-Q}, \hat{V}\right]=0$. In such exceptional cases, because of their symmetries, the external perturbation cannot modulate the coupling Hamiltonian. It leads to failure in dynamical decoupling.

In the interaction frame of $\hat{V}(t)$, we obtain a modulated Hamiltonian,

$$
\hat{\tilde{\mathcal{H}}}_{0}(t)=\sum_{k^{\prime}, k} \sum_{\beta \gamma} d^{\left(k^{\prime}, k\right)} \eta_{\beta, \gamma}(t) \hat{\sigma}_{\beta, k^{\prime}} \hat{\sigma}_{\gamma, k},
$$

where $\eta_{\beta \gamma}(t)=c_{\beta z}(t) c_{\gamma z}(t)-c_{\beta x}(t) c_{\gamma x}(t) / 2-c_{\beta y}(t) c_{\gamma y}(t) / 2$. We defined a cost function for the synthesis of $v_{x}(t)$ and $v_{y}(t)$ in a manner similar to that in Sec. III, excluding $d^{\left(k^{\prime}, k\right)}$ from the cost function to manage the arbitrary strengths of the interactions and considering $\left\|\hat{\mathcal{H}}_{Q-Q} T\right\|<1$. The synthesized waveforms expressed in a Fourier series are listed in Table II and the graphical one is displayed in the top panel of Fig. 2(a). 
TABLE II. Parameters of the DD sequence in Sec. IV.

\begin{tabular}{lcr}
\hline \hline$n$ & $v_{x, n} T / \pi$ & \multicolumn{1}{c}{$v_{y, n} T / \pi$} \\
\hline 1 & -4.8892576 & -2.6291726 \\
2 & -3.1490576 & -3.4112889 \\
3 & -14.317448 & -1.7326439 \\
4 & -0.0929321 & 4.2805093 \\
5 & 6.8394959 & -3.7925374 \\
6 & -0.6645375 & -2.3678092 \\
7 & 0.3344480 & -2.5797746 \\
8 & -1.5042059 & -1.9232075 \\
9 & 2.3863574 & -4.2795712 \\
\hline \hline
\end{tabular}

Here, we emphasize again that it is not necessary to redo the numerical generation for various sets of coupling strengths and the number of qubits.

We evaluated the synthesized DD waveform by simulating a one-dimensional qubit chain, for simplicity, by considering couplings up to the second-nearest qubits, and compared its performance with that of MREV16 [45], which is known to be powerful for this type of coupling. The Hamiltonian of the qubit chain was defined using the coupling strengths in Eq. (14),

$$
d^{\left(k, k^{\prime}\right)}= \begin{cases}\pi / T & \left(k^{\prime}=k+1\right) \\ \pi / 8 T & \left(k^{\prime}=k+2\right) \\ 0 & \text { (otherwise) }\end{cases}
$$

Because it was difficult for us to calculate the gate fidelity defined in Eq. (13) for several qubits, we used the state fidelity for typical quantum states and the trace fidelity defined as $F(\mathcal{C})=\operatorname{Tr}\left[\sqrt{C^{\dagger} C}\right]$ instead, where $C$ is the unitary representation of $\mathcal{C}$. Four states were used and defined as follows: The coherent spin state directed toward the $x$ axis is the eigenstate of the collective $x$ operator $\sum_{k} \hat{\sigma}_{x, k}$ that has the maximum eigenvalue. The GHZ state is $|\mathrm{GHZ}\rangle=|0\rangle^{\otimes n}+|1\rangle^{\otimes n}$, where $n$ is the number of qubits. The maximally entangled state is $|\mathrm{MES}\rangle=2^{-n / 2} \sum_{i=0}^{2^{n}}|i\rangle$. The Dicke state is one of the eigenstates of the collective $z$ operator and the total spin operator $\hat{\sigma} \cdot \hat{\sigma}$ that have the $\hat{\sigma}_{z}$ eigenvalue
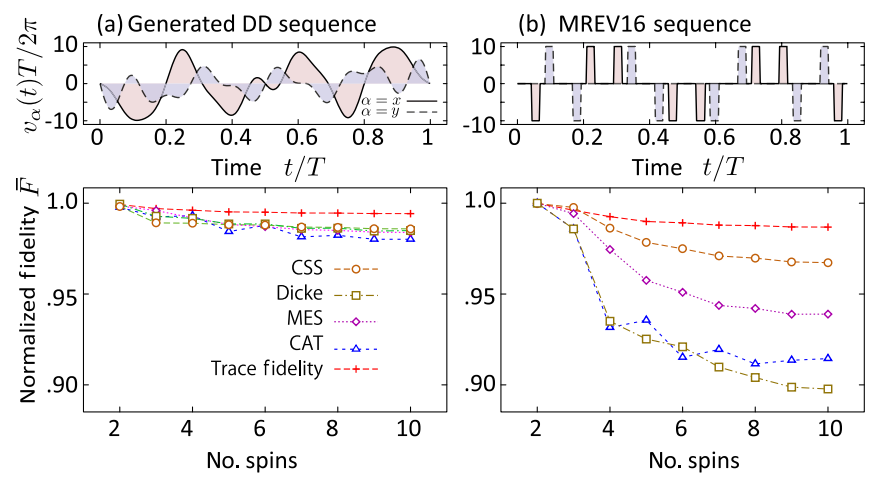

FIG. 2. DD performance as evaluated for an $n$-qubit chain system coupled through dipolar interactions with (a) optimized pulse sequence and (b) MREV16 sequence. Fidelity $F$ is normalized so that $\bar{F}=1-(1-F) /\left\|\hat{\mathcal{H}}_{Q-Q}\right\|$ because $\left\|\hat{\mathcal{H}}_{Q-Q}\right\|$ becomes larger as the number of qubits increases. closest to zero and the maximum $\hat{\sigma} \cdot \hat{\sigma}$ eigenvalue. The energy and peak amplitude of this DD sequence were restricted to $13.8 \pi / T$ and $20 \pi / T$ so that they were almost equal to those of MREV16 (16 $\pi / T$ and $20 \pi / T$, respectively).

Figure 2 shows the fidelity per cycle against the number of qubits in the system. The synthesized sequence effectively decouples qubit-qubit interactions for any state available in this example, whereas MREV16 shows considerable degradation in the nonclassical states owing to cooperative destruction caused by the finite width of the pulses and the qubit-qubit interactions. Given that the numerically synthesized sequence is optimized to isotropically suppress any operator coefficient up to the first order, experimentally, it works better for the nonclassical states, which are necessary for QIP experiments.

\section{PERFORMANCE VERSUS RESOURCES}

Our examples, with smoothly modulated DD sequences, are free from the finite pulse width problem and are robust against distortion in the waveforms under the finite-frequency bandwidth limitation. Available resources can be specified in terms of the maximum control amplitude $\|\hat{V}(t) T\|$ and frequency bandwidth of $\hat{V}(t)$, so that DD sequence synthesis is performed within the available resources. We found that there is a close relationship among the DD performance, available bandwidth, and peak amplitude. Figure 3 shows the imperfection of DD sequences used in Sec. IV under the resource restrictions. The root mean square of the operator coefficients in an average Hamiltonian up to the first order $\sqrt{\Phi^{(0)}+\Phi^{(1)}}$ was used as a measure of imperfection because this value indicates the suppression ratio sum of residual coupling strength at $\left\|\hat{\mathcal{H}}_{0} T\right\| \sim 1$. We imposed a frequency bandwidth limitation on $\hat{V}(t)$ using $p$ in Eq. (12). The bandwidth of waveforms $f_{\mathrm{BW}}$ was defined as $2 p / T$.

The figure clearly shows that the imperfection of the generated DD sequences is monotonically improved with an increase in the available resources; however, it also suggests

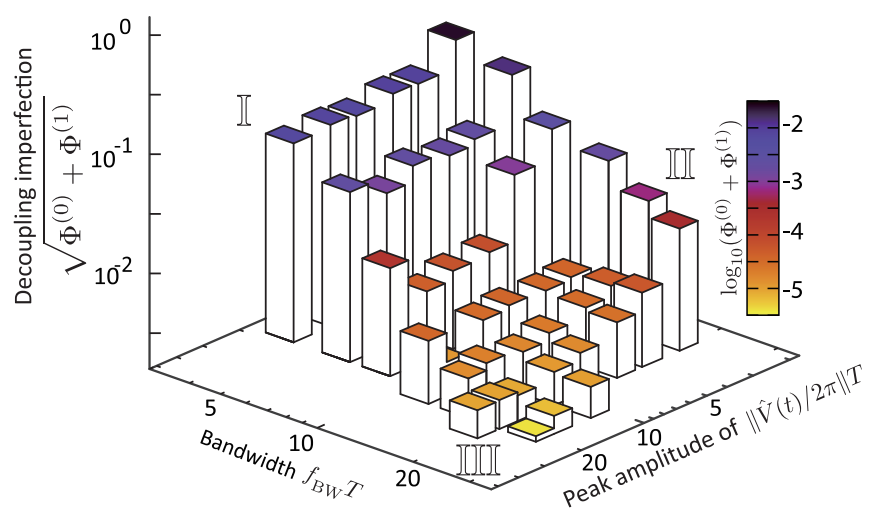

FIG. 3. Decoupling imperfection under resource restrictions in terms of amplitude of $\|\hat{V}(t) T\|$ and bandwidth of $\hat{V}(t)$. Imperfection is investigated with linear combination of zero- and first-order cost functions defined in Sec. IV, so that it is the reciprocal of DD performance. Regions in which the maximum amplitude or the bandwidth is excessively supplied are labeled as I and II, respectively, and the region where both resources are sufficiently supplied is labeled as III. All axes are in logarithmic scale. 


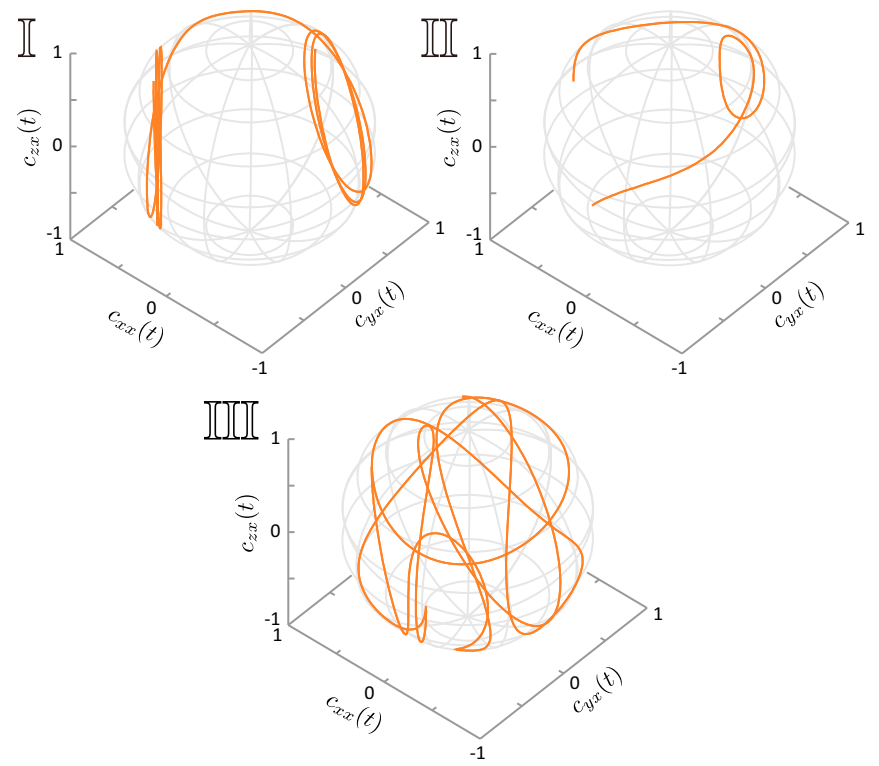

FIG. 4. System-modulation matrices $c_{\alpha x}(t)(\alpha=x, y, z)$ in regions I-III, represented in Cartesian coordinates. $c_{\alpha x}(t)$ are displayed as functions of $t \in[0, T]$.

that there is a trade-off between the minimum attainable imperfection and available resources. As seen from the figure, converged values of the cost function $\sqrt{\Phi^{(0)}+\Phi^{(1)}}$ are closely related to the available resources. This can also be seen in Ref. [32], where the imperfection of synthesized DDs depends on the available amplitude of $\hat{V}(t)$. Although the converged values are not always the global minima of $\Phi(\zeta)$ (because of the existence of local minima in nonlinear minimization), the figure suggests that available resources bound the performance of dynamical decoupling sequences.

The figure shows another relationship between the resources: the decoupling imperfection becomes large when either of the resources is oversupplied. Region I in Fig. 3 is ascribed to over driving, which denotes lack of bandwidth of $\hat{V}(t)$ to utilize the large amount of driving energy for controlling the qubits. This behavior can be seen in region I of Fig. 4, where a part of a system-modulation matrix $c_{\alpha x}(t)$ $(\alpha=x, y, z)$ in the region is represented as a trajectory in Cartesian coordinates. Given that the trajectory needs to have loops to consume the excess driving energy, the trajectory of the system-modulation matrix is constrained from moving efficiently to decouple and, consequently, the performance drops. Region II in Fig. 3 is ascribed to over modulation of $\hat{V}(t)$, wherein the amount of energy supplied for driving the qubits is insufficient (region II in Fig. 4). When both resources are sufficiently supplied to drive and modulate, as in region III in Fig. 3, the system-modulation matrix in the region efficiently averages out unwanted interactions (region III in Fig. 4).

\section{CONCLUSION}

We presented a general framework to numerically synthesize DD sequences, which can tailor them to a given set of qubits, environment, and realistic resource limitations. To this end, a cost function for the numerical synthesis of the DD sequences was built using system-modulation matrices of operators in Lie algebra, instead of a unitary propagator, in order to fully exploit the symmetries that exist in qubits and environments. The effects of finite pulse width are naturally incorporated in the system-modulation matrix. We presented two examples to demonstrate the robustness of the generated DD waveform to flip-angle and phase-orthogonality errors, regardless of the error amplitude, and the capability of the DD waveform to eliminate unwanted qubit-qubit interactions, regardless of the number of qubits with protected nonclassical qubit states. The trade-off between decoupling imperfection and resource limitations was numerically studied. In the near future, we intend to rearrange a system Hamiltonian with the help of an external perturbation as introduced in [27], which is also applicable to QC and QIP experiments.

\section{ACKNOWLEDGMENTS}

This work was supported by the Funding Program for World-Leading Innovative R\&D on Science and Technology (FIRST), "Quantum Information Processing Project" from cabinet office, Japan, Grant-in-Aid for Scientific Research on Innovative Areas "Quantum Cybernetics" (Grant No. JP21102004). Y.T. was supported in part by Grant-in-Aid for JSPS Research Fellow (Grants No. JP10J01531 and No. JP15J10499) from JSPS KAKENHI, Japan.

\section{APPENDIX A: FLOQUET HAMILTONIAN THEORY}

A periodic time-dependent Hamiltonian $\hat{\tilde{\mathcal{H}}}(t)$ can be averaged over a period $T$ using an approach based on the Floquet theory. Consider a Fourier expansion of $\hat{\tilde{\mathcal{H}}}(t)$ such that

$$
\hat{\tilde{\mathcal{H}}}(t)=\sum_{n} \hat{\mathcal{H}}_{\mathrm{F}}^{(n)} \exp (2 i \pi n t / T) .
$$

As stated in [40], average Hamiltonians up to second order are given by

$$
\begin{gathered}
\hat{\mathcal{H}}_{\text {eff }}^{(0)}=\hat{\mathcal{H}}_{\mathrm{F}}^{(0)}, \\
\hat{\mathcal{H}}_{\text {eff }}^{(1)}=\frac{T}{4 \pi} \sum_{n \neq 0} \frac{1}{n}\left[\hat{\mathcal{H}}_{\mathrm{F}}^{(n)}, \hat{\mathcal{H}}_{\mathrm{F}}^{(-n)}\right], \\
\hat{\mathcal{H}}_{\text {eff }}^{(2)}=\frac{T^{2}}{12 \pi^{2}} \sum_{n \neq 0} \sum_{n^{\prime}+n \neq 0} \frac{1+(1 / 2) \delta_{n^{\prime}, 0}}{n\left(n+n^{\prime}\right)} \\
\times\left[\left[\hat{\mathcal{H}}_{\mathrm{F}}^{(n)}, \hat{\mathcal{H}}_{\mathrm{F}}^{\left(n^{\prime}\right)}\right], \hat{\mathcal{H}}_{\mathrm{F}}^{\left(-n-n^{\prime}\right)}\right] .
\end{gathered}
$$

\section{APPENDIX B: DERIVATION OF AVERAGE HAMILTONIAN FOR DEPHASING PROBLEM}

The Hamiltonian of the system $\hat{\mathcal{H}}_{0}$ is considered as $\hat{\mathcal{H}}_{0}=$ $\hat{\mathcal{H}}_{Q-B}+\hat{\mathcal{H}}_{E}$ in the main text, where

$$
\begin{gathered}
\hat{\mathcal{H}}_{Q-B}=\sum_{k} g^{(k)} \hat{\sigma}_{z} \hat{\sigma}_{z, k}, \\
\hat{\mathcal{H}}_{E}=\varepsilon_{1} v_{x}(t) \hat{\sigma}_{x}+\varepsilon_{3} v_{y}(t) \hat{\sigma}_{y} \\
+\varepsilon_{2} v_{y}(t) \hat{\sigma}_{x}+\varepsilon_{4} v_{x}(t) \hat{\sigma}_{y},
\end{gathered}
$$


and $\hat{\sigma}_{\alpha}, \hat{\sigma}_{\alpha, k}(\alpha=x, y, z)$ denote Pauli operators of the qubit and the $k$ th TLS, respectively. A unitary operator $\hat{U}_{V}(t)=$ $\hat{\mathcal{T}} \exp \left[i \int_{0}^{t} V\left(t^{\prime}\right) d t^{\prime}\right]$ modulates the Hamiltonian $\hat{\mathcal{H}}_{0}$ in the interaction frame of $V(t)$ such that

$$
\begin{gathered}
\hat{\tilde{\mathcal{H}}}_{Q-B}(t)=\sum_{\alpha, k, n} g^{(k)} c_{\alpha z, n} \hat{\sigma}_{\alpha} \hat{\sigma}_{z, k} e^{2 \pi i n t / T}, \\
\hat{\tilde{\mathcal{H}}}_{E}(t)=\sum_{\alpha, n}\left\{\varepsilon_{1} \sum_{n^{\prime}} v_{x, n-n^{\prime}} c_{\alpha x, n^{\prime}}+\varepsilon_{2} \sum_{n^{\prime}} v_{y, n-n^{\prime}} c_{\alpha x, n^{\prime}}+\varepsilon_{3} \sum_{n^{\prime}} v_{y, n-n^{\prime}} c_{\alpha y, n^{\prime}}+\varepsilon_{4} \sum_{n^{\prime}} v_{x, n-n^{\prime}} c_{\alpha y, n^{\prime}}\right\} \hat{\sigma}_{\alpha} e^{2 \pi i n t / T},
\end{gathered}
$$

where $v_{\alpha, n}=T^{-1} \int_{0}^{T} v_{\alpha}(t) \exp (-2 \pi i n t / T)$ and $c_{\beta \alpha}(t)=\sum_{n} c_{\beta \alpha, n} \exp (2 \pi i n t / T)=\operatorname{Tr}\left[\hat{U}_{V} \hat{\sigma}_{\alpha} \hat{U}_{V}^{\dagger} \hat{\sigma}_{\beta}\right] / \operatorname{Tr}\left[\hat{\sigma}_{\beta}^{2}\right]$. If we write

$$
\begin{aligned}
& d_{\alpha, n}^{(1)}=\sum_{n^{\prime}} v_{x, n-n^{\prime}} c_{\alpha x, n^{\prime}}, \quad d_{\alpha, n}^{(2)}=\sum_{n^{\prime}} v_{y, n-n^{\prime}} c_{\alpha x, n^{\prime}}, \\
& d_{\alpha, n}^{(3)}=\sum_{n^{\prime}} v_{y, n-n^{\prime}} c_{\alpha y, n^{\prime}}, \quad d_{\alpha, n}^{(4)}=\sum_{n^{\prime}} v_{x, n-n^{\prime}} c_{\alpha y, n^{\prime}},
\end{aligned}
$$

the Fourier series of the modulated Hamiltonians are

$$
\begin{gathered}
\hat{\mathcal{H}}_{Q-B, \mathrm{~F}}^{(n)}=\sum_{\alpha, k} g^{(k)} c_{\alpha z, n} \hat{\sigma}_{\alpha} \hat{\sigma}_{z, k}, \\
\hat{\mathcal{H}}_{E, \mathrm{~F}}^{(n)}=\sum_{\alpha, i} \varepsilon_{i} d_{\alpha, n}^{(i)} \hat{\sigma}_{\alpha} .
\end{gathered}
$$

The zeroth-order average Hamiltonian is easily obtained from $\hat{\mathcal{H}}_{\text {eff }}^{(0)}=\hat{\mathcal{H}}_{Q-B, \mathrm{~F}}^{(0)}+\hat{\mathcal{H}}_{E, \mathrm{~F}}^{(0)}$, and is given by

$$
\hat{\mathcal{\mathcal { H }}}_{\mathrm{eff}}^{(0)}=\sum_{\alpha, k} g^{(k)} c_{\alpha z, 0} \hat{\sigma}_{z} \hat{\sigma}_{z, k}+\sum_{\alpha, i} \varepsilon_{i} d_{\alpha, 0}^{(i)} \hat{\sigma}_{\alpha} .
$$

The first-order average Hamiltonian is obtained from Eq. (A3) and is given by

$$
\begin{aligned}
\hat{\mathcal{H}}_{\text {eff }}^{(1)} & =\frac{T}{4 \pi} \sum_{n \neq 0} \frac{1}{n}\left[\hat{\mathcal{H}}_{\mathrm{F}}^{(n)}, \hat{\mathcal{H}}_{\mathrm{F}}^{(-n)}\right] \\
& =\frac{T}{2 \pi} \sum_{n>0} \frac{1}{n}\left[\hat{\mathcal{H}}_{Q-B, \mathrm{~F}}^{(n)}+\hat{\mathcal{H}}_{E, \mathrm{~F}}^{(n)}, \hat{\mathcal{H}}_{Q-B, \mathrm{~F}}^{(-n)}+\hat{\mathcal{H}}_{E, \mathrm{~F}}^{(-n)}\right] \\
& =\frac{T}{2 \pi} \sum_{n>0} \frac{1}{n}\left\{\left[\hat{\mathcal{H}}_{Q-B, \mathrm{~F}}^{(n)}, \hat{\mathcal{H}}_{Q-B, \mathrm{~F}}^{(-n)}\right]+\left[\hat{\mathcal{H}}_{E, \mathrm{~F}}^{(n)}, \hat{\mathcal{H}}_{E, \mathrm{~F}}^{(-n)}\right]+\left[\hat{\mathcal{H}}_{Q-B, \mathrm{~F}}^{(n)}, \hat{\mathcal{H}}_{E, \mathrm{~F}}^{(-n)}\right]-\left[\hat{\mathcal{H}}_{Q-B, \mathrm{~F}}^{(-n)}, \hat{\mathcal{H}}_{E, \mathrm{~F}}^{(n)}\right]\right\},
\end{aligned}
$$

where

$$
\begin{aligned}
{\left[\hat{\mathcal{H}}_{Q-B, \mathrm{~F}}^{(n)}, \hat{\mathcal{H}}_{Q-B, \mathrm{~F}}^{(-n)}\right] } & =\sum_{k^{\prime}, k} g^{\left(k^{\prime}\right)} g^{(k)} \sum_{\alpha^{\prime}, \alpha} c_{\alpha^{\prime} z, n} c_{\alpha z,-n}\left[\hat{\sigma}_{\alpha^{\prime}}, \hat{\sigma}_{\alpha}\right] \hat{\sigma}_{z, k^{\prime}} \hat{\sigma}_{z, k} \\
& =\sum_{k^{\prime}, k} g^{\left(k^{\prime}\right)} g^{(k)} \sum_{\alpha^{\prime}<\alpha}\left(c_{\alpha^{\prime} z, n} c_{\alpha z,-n}-c_{\alpha z, n} c_{\alpha^{\prime} z,-n}\right)\left[\hat{\sigma}_{\alpha^{\prime}}, \hat{\sigma}_{\alpha}\right] \hat{\sigma}_{z, k^{\prime}} \hat{\sigma}_{z, k} \\
& =\sum_{k^{\prime}, k} g^{\left(k^{\prime}\right)} g^{(k)} \sum_{\alpha^{\prime}<\alpha}\left(c_{\alpha^{\prime} z, n} c_{\alpha z, n}^{*}-c_{\alpha z, n} c_{\alpha^{\prime} z, n}^{*}\right)\left[\hat{\sigma}_{\alpha^{\prime}}, \hat{\sigma}_{\alpha}\right] \hat{\sigma}_{z, k^{\prime}} \hat{\sigma}_{z, k} \\
& =\sum_{k^{\prime}, k} g^{\left(k^{\prime}\right)} g^{(k)} \sum_{\alpha^{\prime}<\alpha} 2 \epsilon_{\alpha^{\prime} \alpha \beta} \operatorname{Im}\left[c_{\alpha z, n} c_{\alpha^{\prime} z, n}^{*}\right] \hat{\sigma}_{\beta} \hat{\sigma}_{z, k^{\prime}} \hat{\sigma}_{z, k}, \\
{\left[\hat{\mathcal{H}}_{E, \mathrm{~F}}^{(n)}, \hat{\mathcal{H}}_{E, \mathrm{~F}}^{(-n)}\right] } & =\sum_{\alpha^{\prime}<\alpha} \sum_{i} \varepsilon_{i}^{2} 2 \epsilon_{\alpha^{\prime} \alpha \beta} \operatorname{Im}\left[d_{\alpha^{\prime}, n}^{(i)} d_{\alpha, n}^{(i) *}\right] \hat{\sigma}_{\beta}+\sum_{\alpha^{\prime}<\alpha} \sum_{i^{\prime}<i} \varepsilon_{i^{\prime}} \varepsilon_{i} 2 \epsilon_{\alpha^{\prime} \alpha \beta} \operatorname{Im}\left[d_{\alpha^{\prime}, n}^{(i)} d_{\alpha, n}^{(i) *}+d_{\alpha^{\prime}, n}^{(i) *} d_{\alpha, n}^{\left(i^{\prime}\right)}\right] \hat{\sigma}_{\beta}, \\
{\left[\hat{\mathcal{H}}_{Q-B, \mathrm{~F}}^{(n)}, \hat{\mathcal{H}}_{E, \mathrm{~F}}^{(-n)}\right] } & =\sum_{k} \sum_{i} g^{(k)} \varepsilon_{i} \sum_{\alpha^{\prime}<\alpha} 2 \epsilon_{\alpha^{\prime} \alpha \beta} \operatorname{Im}\left[c_{\alpha^{\prime} z, n}^{*} d_{\alpha, n}^{(i)}+c_{\alpha z, n} d_{\alpha^{\prime}, n}^{(i) *}\right] \hat{\sigma}_{\beta} \hat{\sigma}_{z, k},
\end{aligned}
$$


and $\epsilon_{\alpha \beta \gamma}$ is the Levi-Civita symbol. $\sum_{\alpha^{\prime}<\alpha}$ represents the summation of $\left(\alpha^{\prime}, \alpha\right)=\{(x, y),(x, z),(y, z)\}$. Here, we used the identity $c_{\alpha \beta,-n}=c_{\alpha \beta, n}^{*}$ because $c_{\alpha \beta}(t)$ is real. The first-order average Hamiltonian then becomes

$$
\begin{aligned}
\hat{\mathcal{H}}_{\mathrm{eff}}^{(1)}= & \frac{T}{\pi} \sum_{k^{\prime}, k} g^{\left(k^{\prime}\right)} g^{(k)} \sum_{\alpha^{\prime}<\alpha} \varepsilon_{\alpha^{\prime} \alpha \beta}\left(\sum_{n>0} \frac{1}{n} \operatorname{Im}\left[c_{\alpha z, n} c_{\alpha^{\prime} z, n}^{*}\right]\right) \hat{\sigma}_{\beta} \hat{\sigma}_{z, k^{\prime}} \hat{\sigma}_{z, k}+\frac{T}{\pi} \sum_{\alpha^{\prime}<\alpha} \sum_{i} \varepsilon_{i}^{2} \epsilon_{\alpha^{\prime} \alpha \beta}\left(\sum_{n>0} \frac{1}{n} \operatorname{Im}\left[d_{\alpha^{\prime}, n}^{(i)} d_{\alpha, n}^{(i) *}\right]\right) \hat{\sigma}_{\beta} \\
& +\frac{T}{\pi} \sum_{\alpha^{\prime}<\alpha} \sum_{i^{\prime}<i} \varepsilon_{i^{\prime}} \varepsilon_{i} \epsilon_{\alpha^{\prime} \alpha \beta}\left(\sum_{n>0} \frac{1}{n} \operatorname{Im}\left[d_{\alpha^{\prime}, n}^{(i)} d_{\alpha, n}^{\left(i^{\prime}\right) *}+d_{\alpha^{\prime}, n}^{(i) *} d_{\alpha, n}^{\left(i^{\prime}\right)}\right]\right) \hat{\sigma}_{\beta} \\
& +\frac{2 T}{\pi} \sum_{k} \sum_{i} g^{(k)} \varepsilon_{i} \sum_{\alpha^{\prime}<\alpha} \epsilon_{\alpha^{\prime} \alpha \beta}\left(\sum_{n>0} \frac{1}{n} \operatorname{Im}\left[c_{\alpha^{\prime} z, n}^{*} d_{\alpha, n}^{(i)}+c_{\alpha z, n} d_{\alpha^{\prime}, n}^{(i) *}\right]\right) \hat{\sigma}_{\beta} \hat{\sigma}_{z, k} .
\end{aligned}
$$

\section{APPENDIX C: DERIVATION OF THE COST FUNCTION FOR DEPHASING PROBLEM}

The zeroth-order cost function $\Phi^{(0)}$ is defined from Eq. (B7) as follows:

$$
\Phi^{(0)}=\sum_{\alpha=x, y, z}\left\{\left|c_{\alpha z, 0}\right|^{2}+w^{2} \sum_{i}\left|d_{\alpha, 0}^{(i)}\right|^{2}\right\} .
$$

The first-order cost function $\Phi^{(1)}$ is defined such that terms within the large parentheses in Eq. (B8) become zero, and is given by

$$
\begin{aligned}
\Phi^{(1)}= & \sum_{\alpha^{\prime}<\alpha}\left|\sum_{n>0} \frac{1}{n} \operatorname{Im}\left[c_{\alpha z, n} c_{\alpha^{\prime} z, n}^{*}\right]\right|^{2}+w^{4} \sum_{\alpha^{\prime}<\alpha} \sum_{i}\left|\sum_{n>0} \frac{1}{n} \operatorname{Im}\left[d_{\alpha^{\prime}, n}^{(i)} d_{\alpha, n}^{(i) *}\right]\right|^{2} \\
& +w^{4} \sum_{\alpha^{\prime}<\alpha} \sum_{i^{\prime}<i}\left|\sum_{n>0} \frac{1}{n} \operatorname{Im}\left[d_{\alpha^{\prime}, n}^{(i)} d_{\alpha, n}^{\left(i^{\prime}\right) *}+d_{\alpha^{\prime}, n}^{(i) *} d_{\alpha, n}^{\left(i^{\prime}\right)}\right]\right|^{2}+w^{2} \sum_{\alpha^{\prime}<\alpha} \sum_{i}\left|\sum_{n>0} \frac{1}{n} \operatorname{Im}\left[c_{\alpha^{\prime} z, n}^{*} d_{\alpha, n}^{(i)}+c_{\alpha z, n} d_{\alpha^{\prime}, n}^{(i) *}\right]\right|^{2},
\end{aligned}
$$

where $w$ is the deliberate weight for the pulse errors set to $1 / 100$.

[1] P. Zanardi, Phys. Lett. A 258, 77 (1999).

[2] L. Viola, E. Knill, and S. Lloyd, Phys. Rev. Lett. 82, 2417 (1999).

[3] K. Khodjasteh and D. A. Lidar, Phys. Rev. Lett. 95, 180501 (2005).

[4] L. Viola, S. Lloyd, and E. Knill, Phys. Rev. Lett. 83, 4888 (1999).

[5] M. S. Byrd and D. A. Lidar, Phys. Rev. A 67, 012324 (2003).

[6] J. R. West, D. A. Lidar, B. H. Fong, and M. F. Gyure, Phys. Rev. Lett. 105, 230503 (2010).

[7] K. Khodjasteh, D. A. Lidar, and L. Viola, Phys. Rev. Lett. 104, 090501 (2010).

[8] H. K. Ng, D. A. Lidar, and J. Preskill, Phys. Rev. A 84, 012305 (2011).

[9] S. Meiboom and D. Gill, Rev. Sci. Instrum. 29, 688 (1958).

[10] W.-K. Rhim, D. D. Elleman, and R. W. Vaughan, J. Chem. Phys. 59, 3740 (1973).

[11] G. S. Uhrig, Phys. Rev. Lett. 98, 100504 (2007).

[12] J. R. West, B. H. Fong, and D. A. Lidar, Phys. Rev. Lett. 104, 130501 (2010).

[13] A. M. Souza, G. A. Álvarez, and D. Suter, Phys. Rev. Lett. 106, 240501 (2011).

[14] M. J. Biercuk, H. Uys, A. P. VanDevender, N. Shiga, W. M. Itano, and J. J. Bollinger, Nature (London) 458, 996 (2009).

[15] X. Peng, D. Suter, and D. A. Lidar, J. Phys. B 44, 154003 (2011).

[16] J. Bylander, S. Gustavsson, F. Yan, F. Yoshihara, K. Harrabi, G. Fitch, D. G. Cory, Y. Nakamura, J.-S. Tsai, and W. D. Oliver, Nat. Phys. 7, 565 (2011).
[17] J. A. Jones and E. Knill, J. Magn. Reson. 141, 322 (1999).

[18] D. W. Leung, I. L. Chuang, F. Yamaguchi, and Y. Yamamoto, Phys. Rev. A 61, 042310 (2000).

[19] M. D. Bowdrey, J. A. Jones, E. Knill, and R. Laflamme, Phys. Rev. A 72, 032315 (2005).

[20] L. M. K. Vandersypen, M. Steffen, G. Breyta, C. S. Yannoni, M. H. Sherwood, and I. L. Chuang, Nature (London) 414, 883 (2001).

[21] K. Khodjasteh and D. A. Lidar, Phys. Rev. A 75, 062310 (2007).

[22] T. Barbara, J. Martin, and J. Wurl, J. Magn. Reson. 93, 497 (1991)

[23] Y. Tabuchi, M. Negoro, K. Takeda, and M. Kitagawa, J. Magn. Reson. 204, 327 (2010).

[24] L. Viola and E. Knill, Phys. Rev. Lett. 90, 037901 (2003).

[25] G. Quiroz and D. A. Lidar, Phys. Rev. A 88, 052306 (2013).

[26] A. P. Peirce, M. A. Dahleh, and H. Rabitz, Phys. Rev. A 37, 4950 (1988).

[27] E. Brion, F. Carlier, G. Harel, and V. M. Akulin, J. Phys. B 44, 154001 (2011).

[28] N. Khaneja, T. Reiss, C. Kehlet, T. Schulte-Herbrüggen, and S. J. Glaser, J. Magn. Reson. 172, 296 (2005).

[29] J. Zhang, D. Gangloff, O. Moussa, and R. Laflamme, Phys. Rev. A 84, 034303 (2011).

[30] T. W. Borneman, M. D. Hürlimann, and D. G. Cory, J. Magn. Reson. 207, 220 (2010).

[31] G. Gordon, G. Kurizki, and D. A. Lidar, Phys. Rev. Lett. 101, 010403 (2008). 
[32] J. Clausen, G. Bensky, and G. Kurizki, Phys. Rev. Lett. 104, 040401 (2010).

[33] G. A. Paz-Silva, S.-W. Lee, T. J. Green, and L. Viola, New J. Phys. 18, 073020 (2016).

[34] J.-M. Cai, B. Naydenov, R. Pfeiffer, L. P. McGuinness, K. D. Jahnke, F. Jelezko, M. B. Plenio, and A. Retzker, New J. Phys. 14, 113023 (2012).

[35] N. C. Jones, T. D. Ladd, and B. H. Fong, New J. Phys. 14, 093045 (2012).

[36] M. D. Grace, J. M. Dominy, W. M. Witzel, and M. S. Carroll, Phys. Rev. A 85, 052313 (2012).

[37] P. Mansfield, M. J. Orchard, D. C. Stalker, and K. H. B. Richards, Phys. Rev. B 7, 90 (1973).

[38] U. Haeberlen and J. S. Waugh, Phys. Rev. 175, 453 (1968).
[39] R. Raussendorf and J. Harrington, Phys. Rev. Lett. 98, 190504 (2007).

[40] I. Scholz, J. D. van Beek, and M. Ernst, Solid State Nucl. Magn. Reson. 37, 39 (2010).

[41] R. Tycko, Phys. Rev. Lett. 51, 775 (1983).

[42] H. G. Krojanski and D. Suter, Phys. Rev. Lett. 93, 090501 (2004).

[43] J. Baugh, O. Moussa, C. A. Ryan, A. Nayak, and R. Laflamme, Nature (London) 438, 470 (2005).

[44] M. Negoro, K. Tateishi, A. Kagawa, and M. Kitagawa, Phys. Rev. Lett. 107, 050503 (2011).

[45] T. D. Ladd, D. Maryenko, Y. Yamamoto, E. Abe, and K. M. Itoh, Phys. Rev. B 71, 014401 (2005). 